\title{
Perfil clínico e funcional de idosos atendidos em centro de referência em um município do Amazonas
}

\author{
Clinical and functional profile of elderly people assisted at a reference center in a \\ municipality in Amazonas
}

Perfil clínico funcional de adultos mayores (viejos) atendidos en un centro de referencia de un municipio de Amazonas

Erika Gomes Alves ${ }^{1}$, Danielly Mota da Silva ${ }^{1}$, Adriana Duarte de Sousa ${ }^{1 \star}$.

\begin{abstract}
RESUMO
Objetivo: Traçar o perfil clínico e funcional de idosos atendidos em um centro de referência em saúde do idoso. Métodos: Trata-se de um estudo transversal, descritivo, retrospectivo e documental, envolvendo 74 prontuários de idosos atendidos em um ambulatório de referência em saúde do idoso, localizado em um município amazonense. Utilizou-se uma ficha de avaliação fisioterapêutica dos usuários, além do Índice de Katz e Índice de Lawton para avaliação da capacidade funcional. Resultados: Os prontuários mostraram um perfil com predomínio de indivíduos considerados idosos jovens, do sexo feminino, caracterizados como independentes tanto pela classificação do índice de Katz, quanto pelo Índice de Lawton, não inseridos no mercado de trabalho, com presença de pelo menos 2 comorbidades e prevalência de doenças reumáticas e traumato-ortopédicas. Conclusão: O perfil do idoso atendido no centro de referência mostra predomínio de idosos jovens, com maior grau de independência funcional e prevalência de doenças reumáticas e traumatoortopédicas, além de outras comorbidades. Determinar as características da população assistida poderá contribuir para o desempenho efetivo de toda a equipe, além da elaboração de medidas preventivas, intervenções efetivas, assim como o planejamento de políticas públicas em saúde do idoso.
\end{abstract}

Palavras-chave: Saúde do idoso, Perfil de saúde, Serviços de saúde para idosos.

\begin{abstract}
Objective: To trace the clinical and functional profile of elderly people seen at a referral center for elderly health. Methods: This is a cross-sectional, descriptive, retrospective and documentary study, involving 74 medical records of elderly people seen in a reference outpatient clinic for elderly health, located in an Amazonian municipality. A physical therapy evaluation form was used for users, in addition to the Katz Index and Lawton Index to assess functional capacity. Results: The medical records showed a profile with a predominance of individuals considered to be young elderly women, characterized as independent both by the classification of the Katz index and the Lawton index, not included in the labor market, with the presence of at least 2 comorbidities and prevalence rheumatic and trauma-orthopedic diseases. Conclusion: The profile of the elderly attended at the referral center shows a predominance of young elderly people, with a higher degree of functional independence and prevalence of rheumatic and traumatic-orthopedic diseases, in addition to other comorbidities. Determining the characteristics of the assisted population may contribute to the effective performance of the entire team, in addition to the development of preventive measures, effective interventions, and the planning of public health policies for the elderly.
\end{abstract}

Keywords: Health of the elderly, Health profile, Health services for the aged.

${ }^{1}$ Centro Universitário do Norte (UNINORTE), Manaus - AM. *E-mail: adrianaduartedesousa@hotmail.com 


\section{RESUMEN}

Objetivo: Rastrear el perfil clínico y funcional de adultos mayores atendidos en un centro de referencia de salud de ancianos. Métodos: Estudio transversal, descriptivo, retrospectivo y documental, que involucra 74 registros médicos de adultos mayores atendidas en la clínica ambulatoria para la salud del anciano, ubicado en un municipio amazónico. Se utilizó un formulario de evaluación de fisioterapia para los usuarios, además del Índice Katz y el Índice Lawton para evaluar la capacidad funcional. Resultados: Los registros médicos mostraron un perfil con predominio de individuos considerados mujeres jóvenes de edad avanzada, caracterizados como independientes tanto por la clasificación del índice de Katz como del índice de Lawton, no incluidos en el mercado laboral, con la presencia de al menos 2 comorbilidades y prevalencia. enfermedades reumáticas y traumatológicas-ortopédicas. Conclusión: El perfil de los ancianos atendidos en el centro de referencia muestra un predominio de ancianos jóvenes, con mayor grado de independencia funcional y prevalencia de enfermedades reumáticas y traumático-ortopédicas, además de otras comorbilidades. Determinar las características de la población asistida puede contribuir al desempeño efectivo de todo el equipo, además del desarrollo de medidas preventivas, intervenciones efectivas, así como la planificación de políticas de salud pública para los ancianos.

Palabras clave: Salud del anciano, Perfil de salud, Servicios de salud para ancianos.

\section{INTRODUÇÃO}

Em todo o mundo, o número de idosos tem aumentado drasticamente como um reflexo do aumento da expectativa de vida e da queda das taxas de fertilidade. As melhorias socioeconômicas contribuíram para esse processo, aumentando as chances de sobreviver em idades mais jovens e, consequentemente, de chegar à longevidade. De acordo com a Organização Mundial de Saúde (OMS), o Brasil apresenta projeções para 2025 de cerca de 30 milhões de pessoas com idade igual ou superior a 60 anos (OMS, 2005).

Em Manaus, os idosos representam 6\% da população do município. Comparando-se os anos de 2000 e 2010, houve um aumento de $39,6 \%$ nessa população, o que deixou a capital na $7^{\text {a }}$ posição de cidade brasileira mais populosa e maior cidade da Região Norte do país. Dos 108.902 idosos residentes em Manaus, 56,2\% são mulheres (DATASUS, 2013; IBGE, 2013).

O envelhecimento humano caracteriza-se por diversas alterações morfofisiológicas, que ocorrem de forma progressiva, podendo repercutir no declínio dos diferentes sistemas corporais e estado funcional. Se por um lado o envelhecimento populacional trouxe os benefícios de uma maior longevidade, por outro aumentou a ocorrência do perfil de morbimortalidade, caracterizado por um acréscimo de doenças crônico-degenerativas (VERAS RP, 2012).

Apesar da elevada presença de comorbidades, é possível que este indivíduo leve a vida com autonomia e independência, sendo a funcionalidade um termo importante no que se refere à saúde do idoso. A capacidade funcional pode ser definida como eficiência do idoso em corresponder às demandas físicas do cotidiano, que compreende desde as atividades básicas para uma vida independente até as ações mais complexas da rotina diária (SHUBERT TE, et al., 2006; OKUMA SS, 1997).

$\mathrm{Na}$ velhice costuma-se observar baixos níveis de capacidade funcional, principalmente devido à depreciação das funções físicas, como a diminuição da função dos sistemas osteomuscular, cardiorrespiratório e nervoso, situação que pode impedir os idosos de realizar suas atividades cotidianas com eficiência (SPIRDUSO WW, 2005).

A assistência à saúde da população idosa desafia os modelos de cuidado na medida em que a sociedade envelhece. Os avanços da tecnologia e da ciência da saúde oferecem àqueles que utilizam as modernas ferramentas para a manutenção da saúde a chance de viver mais e em melhores condições (VERAS R, 2011). A avaliação do nível de capacidade funcional dos idosos pode indicar as intervenções direcionadas a essa população, pois é ponto essencial para determinação do risco de dependência futura, da complicação ou instalação de doenças crônicas, de probabilidade de quedas e de índices de morbidade e mortalidade (SHUBERT TE, et al., 2006; OKUMA SS, 1997). 
Pesquisas que avaliam a capacidade funcional tornam-se fundamentais para a mensuração da saúde do idoso, uma vez que se propõem a analisar a habilidade com que o idoso desempenha algumas atividades do seu cotidiano. Assim, a saúde da pessoa idosa relaciona-se às questões de independência funcional, que por sua vez é mensurada através de avaliações funcionais (GUIMARÃES RM e CUNHA UGV, 2004; COSTA MFL, et al., 2003).

Oliveira $H$ (2011), pontua que a caracterização das condições de saúde dos idosos requer informações detalhadas sobre diferentes aspectos da vida desses indivíduos. Nos países desenvolvidos, é crescente o número de investigações que abordam as associações entre a saúde dos idosos e os determinantes demográficos e socioeconômicos, doenças crônicas e a capacidade funcional.

Assim, o conhecimento sobre o perfil desta população torna-se importante para o planejamento das ações de saúde, de forma a oferecer serviços especializados, de acordo com as necessidades da população assistida. Neste sentido, o presente estudo tem como objetivo traçar o perfil epidemiológico e funcional de pacientes atendidos no setor de fisioterapia de um centro especializado em saúde do idoso em um município do Amazonas.

\section{MÉTODOS}

Trata-se de um estudo transversal, descritivo, retrospectivo e documental, envolvendo 74 prontuários de idosos atendidos em um ambulatório de referência em saúde do idoso, localizado em um município amazonense. Esses centros destinam-se ao atendimento ambulatorial de idosos, e existem três unidades espalhadas nas principais zonas da cidade com mais de 75 mil idosos cadastrados. As unidades dispõem de atendimento na área de geriatria, ortopedia, gastroenterologia, ginecologia, clínica médica, odontologia e fisioterapia.

A amostra foi selecionada por conveniência, feita após o cálculo amostral. Foram incluídos prontuários de indivíduos de ambos os sexos, com 60 anos ou mais, que estivessem cadastrados no referido centro de saúde, que tivessem sido atendidos por um período $\geq 30$ dias, entre julho a dezembro de 2017. Foram préselecionados 84 prontuários sendo excluídos aqueles ilegíveis, que não continham informações completas em 3 ou mais itens, com período de tratamento inferior a 30 dias ou que realizaram apenas avaliação fisioterapêutica. Sendo assim, a amostra final foi composta por 74 prontuários.

A coleta de dados ocorreu de julho a dezembro de 2017 através dos prontuários e ficha de avaliação fisioterapêutica dos usuários que se encontravam no Serviço de Arquivo Médico e Estatística (Same) do centro de saúde. Foi utilizado um formulário elaborado pelas próprias pesquisadoras para esse fim, que constou dos seguintes itens: Aspectos sociodemográficos: zona do domicílio, idade, sexo, cor, escolaridade, estado civil, atividade laboral e tamanho da família; Aspectos biológicos e de saúde física: autopercepção da saúde, presença de patologias (diabetes melitus, hipertensão arterial sistêmica, doenças pulmonares, fratura de quadril, reumatismo, déficit visual e auditivo, câncer e outras), medicamentos em uso contínuo e número de internações hospitalares no último ano.

Para avaliação da capacidade funcional, foram utilizadas duas escalas de avaliação das atividades da vida diária (AVD's): Índice de Katz, para atividades básicas da vida diária (ABVD's), desenvolvida para a avaliação dos resultados de tratamentos em idosos e predizer o prognóstico nos doentes crônicos. Consta de seis itens que medem o desempenho do indivíduo nas atividades de autocuidado, os quais obedecem a uma hierarquia de complexidade, da seguinte forma: alimentação, controle de esfíncteres, transferência, higiene pessoal, capacidade para se vestir e tomar banho (ESBENSEN BA, et al., 2006; AVILA JA, et al., 2006).

Índice de Lawtow, para atividades instrumentais da vida diária (AIVD's), avalia oito tarefas necessárias para a vida independente na comunidade: usar o telefone, utilizar transporte, fazer compras, preparar refeições, arrumar a casa, fazer trabalhos manuais domésticos, manusear medicamentos e cuidar das finanças. Para cada tarefa, há três possibilidades de resposta com valores de pontuação de 1 a 3 (1 dependência; 2 - capacidade de realizar a tarefa com ajuda; e 3 - independência). A pontuação final é alcançada pela soma de pontos dos oito domínios e varia de 8 a 24, de tal forma que quanto maior for a pontuação, mais independente será o indivíduo para executar a atividade (LAWTON MP e BRODY EM, 1969; SANTOS RL e VIRTUOSO JSJ, 2008). 
Todas as informações coletadas foram tabuladas em um banco de dados distribuído em planilhas eletrônicas. Foi realizada a análise descritiva dos dados atarvés do software Epi Info 6.0, que viabilizou a construção de tabelas e gráficos, considerando a tendência e a variabilidade de cada variável.

Preliminarmente, foi obtido o consentimento formal do diretor da unidade para compor o protocolo de pesquisa e aprovação pelo Comitê de Ética em Pesquisa (CEP) do Centro Universitário do Norte (UNINORTE), por meio do Parecer ํㅜ 2.352.375/17, atendendo à Resolução oㅡ 466/12, do Conselho Nacional de Saúde (CNS).

\section{RESULTADOS}

A amostra do estudo foi predominantemente composta por sujeitos do sexo feminino $(71,6 \%)$. No que diz respeito à faixa etária, 55,4\% encontravam-se entre 60 a 69 anos, 35,1\% entre 70 a 79 e 9,5\% em idade igual ou superior a 80 anos. Quanto à ocupação profissional $55,4 \%$ informaram não exercer atividades profissionais (Tabela 1).

Tabela 1 - Caracterização dos idosos atendidos no centro de referência, $n=74$.

\begin{tabular}{ccc}
\hline Variável & N & $\%$ \\
\hline Sexo & 53 & 71,6 \\
Feminino & 21 & 28,4 \\
Masculino & & \\
\hline Faixa etária & 41 & 55,4 \\
$60-69$ anos & 26 & 35,1 \\
$70-79$ anos & 7 & 9,5 \\
$\geq 80$ & & \\
\hline Ocupação profissional & 41 & 55,4 \\
Não & 33 & 44,6 \\
\hline Sim & 74 & 100
\end{tabular}

Fonte: ALVES EG, et al., 2020.

Quanto ao diagnóstico clínico, 47,9\% apresentaram doenças reumáticas, 32,9\% traumato-ortopédicas e 19,2\% neurológicas. A ocorrência de quedas no último ano foi de $24,3 \%$, com predomínio entre as mulheres. Em relação à capacidade funcional, 10,3\% apresentaram dependência para atividades básicas de vida diária e $15,3 \%$ e 8,3 apresentaram dependência parcial e total para atividades instrumentais de vida diária, respectivamente (Tabela 2).

Tabela 2 - Condições de saúde e capacidade funcional dos idosos atendidos no centro de referência, n=74.

\begin{tabular}{ccc}
\hline Variáveis & $\mathbf{N}$ & $\%$ \\
\hline Traumato - Ortopédicas & 24 & 32,9 \\
Neurológicas & 14 & 19,2 \\
\hline Comorbidades & & 41,5 \\
\hline Hipertensão arterial sistêmica (HAS) & 17 & 31,7 \\
Diabetes + HAS & 13 & 14,6 \\
Cardiopatias + HAS & 6 & 12,2 \\
Outras & 5 & 42,1 \\
\hline Número de Comorbidades & 32 & 52,6 \\
\hline 0 & 40 & 5,3 \\
\hline 1 a 2 & 4 & 89,7 \\
\hline Capacidade Funcional ABVDs & & \\
\hline Independentes & & 10,3 \\
Dependentes & 66 & 76,4 \\
\hline Capacidade Funcional AIVDs & & 15,3 \\
Independente & & 8,3 \\
\hline Dependência Parcial & 55 &
\end{tabular}

Legenda: *Atividades básicas de vida diária. *Atividades instrumentais de vida diária.

Fonte: ALVES EG, et al., 2020. 


\section{DISCUSSÃO}

Diante dos resultados observou-se maior percentual de indivíduos do sexo feminino, mais jovens e com capacidade funcional preservada. O predomínio de mulheres na população idosa relaciona-se a menor exposição a fatores de risco, como tabagismo e etilismo, além de diferenças comportamentais entre homens e mulheres, maiores cuidados em saúde e menor prevalência de doenças letais. O resultado também revela que, no Brasil, o número de mulheres idosas é maior que o de homens. Tais resultados justificam-se devido as taxas de mortalidade entre os gêneros, característica bastante presente na população brasileira (PACHECO RO e SANTOS SSC; 2004; DAVIM RMB, et al., 2004).

No presente estudo, a variável sexo não apresentou associação estatística quando relacionada com algum grau de dependência, embora tenha sido observada uma maior prevalência de algum grau de dependência entre as mulheres. Os estudos de Brandão DMS, et al. (2009); Del Duca GF, et al. (2009), também apontam para resultados semelhantes, pois encontraram capacidade funcional similar para ambos os sexos. Rosa TEC, et al. (2003), também não confirmaram associação entre sexo e dependência funcional, mas apontaram que o sexo está fortemente associado à ocorrência da dependência, sendo a chance de ocorrer entre as mulheres duas ou mais vezes maior.

Outros estudos como o de Fiedler MRM e Peres KG (2008) e o de Nunes DP, et al. (2010), demonstraram associação em ser do sexo feminino e apresentar dependência. Uma possível explicação para essa associação seria o fato das mulheres possuírem maior expectativa de vida, e maiores chances de desenvolver doenças crônicas incapacitantes.

Também não foi verificada relação estatisticamente significante entre a capacidade funcional e a faixa etária, uma vez que a população do estudo, em sua maioria, era composta por idosos jovens. Trabalhos anteriores mostraram que o avanço da idade, sobretudo em indivíduos com idade igual ou superior a 70 anos, estava associado a uma capacidade funcional considerada inadequada/ com autonomia diminuída (FIEDLER MRM e PERES KG, 2008; BRANDÃO DMS, et al., 2009; DEL DUCA GF, et al., 2009; CARDOSO JH e COSTA JSD, 2010).

Este fato tem explicação pela própria característica do processo de envelhecimento, uma vez que ele está associado ao declínio da função orgânica, promovendo uma diminuição na qualidade e quantidade das informações necessárias para um controle motor e cognitivo eficaz. Agravos à saúde que ocorram nos sistemas sensorial, neurológico e musculoesquelético podem colocar certos indivíduos em risco de desenvolverem restrições funcionais. (NUNES DP, et al., 2010; MACIEL ACC e GUERRA RO, 2007).

Quanto à ocupação profissional, a maioria afirmou estar inativo no mercado de trabalho. Estudos apontam que a principal fonte de renda entre os idosos brasileiros é composta por aposentadorias e pensões (RAMOS LR, 2003; FARIAS-ANTUNEZ S, et al., 2018).

Estudos de base populacional demonstrou que indivíduos idosos que possuíam melhores condições sociais e econômicas também apresentam condições de saúde elevadas (ALVES LC, et al., 2010; GIACOMIN KC, et al., 2005). Em contrapartida, outros estudos revelaram que a associação entre as condições de saúde e os indicadores socioeconômicos não são significativos quando analisados entre a população idosa (BARBOSA KTF, et al., 2017).

$\mathrm{Na}$ amostra estudada, a maioria dos entrevistados (89,7\%), foi classificada como independente pela classificação do índice de Katz. Este resultado está em concordância com estudos transversais realizados em outras parcelas da população nacional. Maciel ACC e Guerra RO (2007), utilizando o mesmo instrumento de avaliação e com uma amostra de 310 idosos de uma área urbana da cidade de Santa Cruz- RN, verificaram que $86,9 \%$ destes também foram classificados como independentes. Pesquisa realizada na cidade de São Paulo demonstrou que o comprometimento no desempenho das atividades básicas da vida diária esteve associado à idade mais avançada e sexo feminino.

Estudo com indivíduos com idade a partir de 60 anos, residentes na zona urbana do município de Pelotas, no Rio Grande do Sul, mostrou maiores prevalências de incapacidade funcional em idosos $\geq 80$ anos, sendo $36,1 \%$ de dependentes para atividades básicas, 34,0\% para instrumentais e 18,1\% para ambas (FARIAS- 
ANTUNEZ S, et al., 2018). A perda da capacidade funcional está associada à predisposição de fragilidade, dependência, institucionalização, risco aumentado de quedas, morte e problemas de mobilidade, trazendo complicações ao longo do tempo, e gerando cuidados de longa permanência e alto custo (REIS LA e DE VASCONCELOS TORRES G, 2011).

Alguns achados relacionam a ocorrência de independência nas ABVD a algumas características como idosos com planos de saúde privado, nível salarial mais alto e maior nível de escolaridade, o que não pode ser atribuído à nossa amostra (SOUSA L, et al., 2003; CARDOSO JH e COSTA JSD, 2010). Outro aspecto que merece ser considerado é que as dimensões avaliadas pelo índice de Katz correspondem a funções elementares à sobrevivência do indivíduo, encontrando-se uma maior prevalência de dependência quando avaliadas tarefas mais complexas, como controlar finanças, utilizar meios de transportes e usar o telefone (MACIEL ACC e GUERRA RO, 2007).

Em relação às Atividades Instrumentais de Vida Diária averiguamos que $76,4 \%$ dos idosos são independentes, $15,3 \%$ necessitam de auxílio e $8,3 \%$ são completamente incapazes na realização de algumas dessas atividades. Concordando com Okuma SS (2001), as Atividades Instrumentais de Vida Diária são mais complexas que as Atividades de Vida Diária. Daí a maior dificuldade apresentada pelos idosos nas AIVDs que nas AVDs. Spector WD, et al. (1987), também destacou que as pessoas idosas provavelmente são mais dependentes nas AIVDs, corroborando com os dados encontrados em nosso estudo.

No Brasil, existe uma carência de pesquisas que analisem isoladamente cada grupo de atividades de vida diária. Geralmente as pesquisas avaliam as tarefas em forma de blocos, o que acaba dificultando um maior aprofundamento da temática. É sabido que idosos residentes em comunidades são considerados mais ativos, o que lhes confere um nível funcional superior, quando comparados aos residentes em instituições de longa permanência (ILP). Restando saber em que níveis estão situados os idosos residentes nas zonas rurais do país, sobretudo das regiões Norte e Nordeste.

Entender o comportamento funcional dentro de cada grupo pode facilitar a compreensão dos fatores envolvidos no declínio funcional e servir como fonte de informação para as ações específicas para cada situação, pois o tipo de ajuda necessária, as demandas de serviços e os custos decorrentes das incapacidades são muito diversas (BARBOSA AR, et al., 2005).

Da mesma forma, estudos que abordem a capacidade funcional nas regiões rurais brasileiras ainda são escassos, mas de maneira geral, avaliando-se os itens das escalas observa-se uma similaridade entre eles e o presente estudo (PARAHYBA MI, et al., 2005; ROSA TEC, et al., 2003). As pequenas discrepâncias encontradas podem ser conferidas devido ao local de realização da pesquisa, aos tipos de escalas utilizadas, à maneira como é realizada a coleta de dados e ao tempo de realização da pesquisa.

Após análise do diagnóstico clínico mais prevalente, percebeu-se a elevada proporção de doenças reumáticas e traumato-ortopédicas. O reumatismo é uma doença comum entre idosos, pois ocorre envelhecimento do aparelho locomotor. Em pessoas idosas existe uma maior incidência de doenças reumáticas, como, por exemplo, a osteoartrose, a osteoporose, a polimialgia reumática, as doenças da coluna, as deficiências de vitamina $D$, entre outras (HOLICK MF, 2007).

Doenças traumato-ortopédicas como a artrose e a artrite, podem interferir na mobilidade da pessoa idosa, limitando-a quanto ao desempenho de atividades diárias, devido ao próprio processo degenerativo patológico, bem como ao quadro álgico que provocam (ARAÚJO LA e BACHION MM, 2005). Estudo realizado GoiâniaGO com 75 idosos mostrou que $44 \%$ da amostra apresentava limitação para deambular, caracterizando interferência na AIVD; $28 \%$ apresentaram déficit no autocuidado para alimentação; 25,35\%, déficit no autocuidado para higiene, e 18,6\%, déficit no autocuidado para vestir-se e arrumar-se, sendo essas tarefas caracterizadas como ABVDs (ARAÚJO LA e BACHION MM, 2005; SHIGUEMOTO GE, 2007).

Para Amaral ACS, et al. (2004) e Almeida MF, et al. (2002), as doenças crônicas apresentam-se como a causa principal de incapacidade, uma razão importante para a demanda dos serviços de saúde, além de responsáveis por vultosos gastos efetuados no setor de saúde, sobretudo para atendimento de idosos, uma vez que os tratamentos prolongados e a recuperação mais lenta e complicada é onerosa para os serviços de saúde. 
Neste contexto, o conceito de morbidade vem sendo utilizado como forma de caracterizar um conjunto de casos de uma mesma patologia ou a soma de agravos de saúde que atingem um determinado grupo de indivíduos, em tempo e lugar específico. Segundo Minari FC (1997), também se considera que a ocorrência, frequência e desvio de bem estar estão associados ao termo morbidade. Quando associadas ao próprio envelhecimento, as morbidades podem resultar em perdas das funções físicas e mentais, interferindo assim na capacidade funcional. Assim, o número de comorbidades é um fator fortemente associado às incapacidades funcionais, bem como à dependência de cuidados em saúde (RAMOS LR, 2003; ROSA TEC, et al., 2003). Estudos demonstram associações importantes entre doenças crônicas e incapacidade funcional em idosos (ROSA TEC, et al., 2003).

$\mathrm{Na}$ amostra estudada, $52,6 \%$ dos idosos eram portadores de pelo menos 2 comorbidades e 5,3\% possuíam mais de duas, sendo a hipertensão arterial sistêmica a comorbidade mais frequente, estando presente em $41,5 \%$ da amostra. A estreita relação entre hipertensão arterial e envelhecimento pode ser justificada por modificações estruturais. Os grandes vasos e as arteríolas aumentam sua espessura da parede, com redução da sua luz, pois há aumento do componente colágeno e diminuição do componente elástico reduzindo a capacidade do funcionamento eficiente (GAZONI FM, et al., 2009). No entanto, quando tratada corretamente, a hipertensão arterial não chega a afetar a capacidade funcional de seus portadores.

Destaque-se também o fato de que $42,1 \%$ dos idosos não apresentavam nenhuma comorbidade, ou seja, foram considerados saudáveis. Segundo a OMS (2014), resultados como esses têm sido alvo de diversos estudos, devendo ser encarados através de um panorama mais amplo, resultado de um trabalho intersetorial e interdisciplinar de promoção de modos de vida saudável.

Com base no que foi exposto, ressalta-se que a amostra deste estudo é um fator limitante, pois não caracteriza uma representatividade de dados populacionais brasileiros, bem como possivelmente contribuiu para divergir de fatores associados ao perfil clínico- funcional encontrados em outras pesquisas nacionais.

Apesar das de alguns resultados particularmente favoráveis tenham sido descritos na pesquisa, os estudos transversais requerem algumas precauções na análise das associações encontradas, em função da impossibilidade de manter relações de causalidade entre a variável desfecho e as exposições. Com isto, o tipo de análise neste estudo não teve como objetivo definir as variáveis envolvidas na gênese do perfil clínicofuncional dos prontuários avaliados, mas sim contribuir na ampliação da discussão sobre as condições de saúde e capacidade funcional dos idosos brasileiros, principalmente daqueles residentes em regiões que apresentam fragilidades sociais conhecidas.

\section{CONCLUSÃO}

Os idosos usuários do serviço de fisioterapia do Centro Especializado apresentam um perfil com predomínio de idosos jovens, do sexo feminino com maior grau de independente funcional e prevalência de doenças reumáticas e traumato-ortopédicas. $\mathrm{O}$ aumento do número de idosos na população implica em maior demanda por serviços em saúde e consequente aumento das despesas com tratamentos em todos os níveis de atenção à saúde. Neste sentido, determinar as características da população assistida poderá contribuir para o desempenho efetivo de toda a equipe, proporcionando a formulação de hipóteses causais, além da elaboração de medidas preventivas, intervenções efetivas, assim como, o planejamento de políticas públicas em saúde do idoso.

\section{REFERÊNCIAS}

1. ALMEIDA MF, et al. Prevalência de doenças crônicas autorreferidas e utilização de serviços de saúde, PNAD/1998, Brasil. Ciênc. Saúde Coletiva, 2002; 7(4): 743-756.

2. ALVES LC, et al. Fatores associados à incapacidade funcional dos idosos no Brasil: análise multinível. Rev. Saúde Pública, 2010; 44(3): 468-478.

3. AMARAL ACS, et al. Perfil de morbidade e de mortalidade de pacientes idosos hospitalizados. Cad. Saúde Pública, $2004 ; 20(6): 1617-1626$.

4. ARAÚJO LA, BACHION MM. Nursing diagnoses of the pattern of mobility in the elderly attended by the Family Health Program. Rev Esc Enferm USP, 2005; 39(1): 53-61. 
5. AVILA JA, et al. Relationship between determining factors for depressive symptoms and for dietary habits in older adults in Mexico. Rev Panam Salud Pública, 2006; 19: 321-30.

6. BARBOSA AR, et al. Functional limitations of Brazilian elderly by age and gender differences: data from SABE Survey. Cad Saúde Pública, 2005; 21: 177-85.

7. BARBOSA KTF, et al. Envelhecimento e vulnerabilidade individual: um panorama dos idosos vinculados a estratégia saúde da família. Texto contexto - enferm, 2017; 26(2): 2700015.

8. BRANDÃO DMS, et al. Capacidade funcional e qualidade de vida em pacientes idosos com ou sem disfagia após acidente vascular encefálico isquêmico. Rev Assoc Med Bras, 2009; 55(6): 738- 743.

9. CARDOSO JH, COSTA JSD. Características epidemiológicas, capacidade funcional e fatores associados em idosos de um plano de saúde. Ciência \& Saúde Coletiva, 2010; 15(6): 2871-2878.

10. COSTA MFL, et al. Condições de saúde, capacidade funcional, uso de serviços de saúde e gastos com medicamentos da população idosa brasileira: um estudo descritivo baseado na Pesquisa Nacional por Amostra de Domicílios. Cad Saúde Pública, 2003; 19(3):735-43.

11. DATASUS. Banco de dados do SUS. Informações sobre saúde. Estatísticas demográficas e socioeconômicas. Disponível em: https://datasus.saude.gov.br/. Acesso em: 16 jan. 2020.

12. DAVIM RMB, et al. Estudo com idosos se instituições asilares no município de Natal/RN: características socioeconômicas e de saúde. Rev. Latino-Am. Enfermagem, 2004; 12(3): 518-24.

13. DEL DUCA GF, et al. Incapacidade funcional para atividades básicas e instrumentais da vida diária em idosos. Rev Saúde Pública, 2009; 43(5): 796- 805.

14. FARIAS-ANTUNEZ S, et al. Incapacidade funcional para atividades básicas e instrumentais da vida diária: um estudo de base populacional com idosos de Pelotas, Rio Grande do Sul, 2014. Epidemiol. Serv. Saúde, 2018; $27(2): 2017290$.

15. FIEDLER MRM, PERES KG. Capacidade funcional e fatores associados em idosos do Sul do Brasil: um estudo de base populacional. Cad. Saúde Pública, 2008; 24(2): 409-415.

16. GAZONI FM, et al. Hipertensão sistólica no idoso. Rev Bras Hipertens, 2009; 16(1): 34-37.

17. GIACOMIN KC, et al. Projeto Bambuí: um estudo de base populacional da prevalência e dos fatores associados à necessidade de cuidador entre idosos The Bambuí Health and Aging Study (BHAS). Cad. Saúde Pública, 2005; 21(1): 80-91.

18. GUIMARÃES RM, CUNHA UGV. Sinais e sintomas em geriatria. São Paulo: Atheneu; 2004.

19. HOLICK MF. Vitamin D deficiency. New England Journal of Medicine, 2007; 357(3): 266-281.

20. IBGE. Instituto Brasileiro de Geografia e Estatística. Censo demográfico de 2010. Rio de Janeiro: Instituto Brasileiro de Geografia e Estatística. Disponível em: https://www.ibge.gov.br/. Acesso em: 15 jan. 2020.

21. LAWTON MP, BRODY EM. Assessment of older people: self-maintaining and instrumental activities of daily living. Gerontologist, 1969; 9(3): 179-86.

22. MACIEL ACC, GUERRA RO. Influência dos fatores biopsicossociais sobre a capacidade funcional de idosos residentes no nordestes do Brasil. Rev Bras Epidemiol, 2007; 10(2): 178-189.

23. MINARI FC. Morbidade: Um Estudo Conceitual e Empírico. Dissertação (Mestrado em Saúde Pública) - Departamento de Epidemiologia, Faculdade de Saúde Pública. Universidade de São Paulo, São Paulo, 1997.

24. NUNES DP, et al. Influência das características sociodemográficas e epidemiológicas na capacidade funcional de idosos residentes em Ubá, Minas Gerais. Ciência \& Saúde Coletiva, 2010; 15(6): 2887-2898.

25. OLIVEIRA H, et al. Perfil epidemiológico de idosos frequentadores de grupos de convivência no município de Iguatu, Ceará. Revista Brasileira de Geriatria e Gerontologia, 2011; 14(1): 123-133.

26. ORGANIZAÇÃO MUNDIAL DA SAÚDE. Envelhecimento ativo: uma política de saúde, 2014.

27. OKUMA SS. O significado da atividade física para o idoso: um estudo fenomenológico. Tese - Instituto de Psicologia. Universidade de São Paulo, São Paulo, 1997; 376p.

28. OKUMA SS. Velhice bem-sucedida: atualizando o conceito de velhice. Temas em Educação Física Adaptada. Revista SOBA-MA, 2001; 64-73.

29. PACHECO RO, SANTOS SSC. Avaliação global de idoso em unidades de PSF. Textos Envelhecimento, 2004; 7(2): 45-61.

30. PARAHYBA MI, et al. Incapacidade funcional entre as mulheres idosas no Brasil. Rev Saúde Pública, 2005; 39: 38391.

31. RAMOS LR. Fatores determinantes do envelhecimento saudável em idosos residentes em centro urbano: Projeto Epidoso, São Paulo. Cadernos de Saúde Pública, 2003; 19: 93-797.

32. REIS LA, DE VASCONCELOS TORRES G. Influência da dor crônica na capacidade funcional de idosos institucionalizados. Revista Brasileira de Enfermagem, 2011; 64(2): 274-280.

33. ROSA TEC, et al. Fatores determinantes da capacidade funcional entre idosos. Rev. Saúde Pública, 2003; 37(1): 4048.

34. SANTOS RL, VIRTUOSO JSJ. Confiabilidade da versão brasileira da escala de atividades instrumentais da vida diária. Rev Bras Promoç Saúde, 2008; $21(4)$ : 290-6.

35. SHIGUEMOTO GE. Avaliação do idoso: anamnese e exame da performance física. In: Rebelatto JR, Morelli JGS, organizadores. Fisioterapia geriátrica - a prática de assistência ao idoso. Barueri: Manole, 2007; 95-97p.

36. SHUBERT TE, et al. Are scores on balance screening tests associated with mobility in older adults? J Geriatr Phys Ther, 2006; 29(1): 33-9.

37. SOUSA L, et al. Qualidade de vida e bem-estar dos idosos: um estudo exploratório na população portuguesa. Rev Saúde Pública, 2003; 37(3): 364-371.

38. 37. SPECTOR WD, et al. The hierarchical relationship between ADL and IADL. J Chron Dis, 1987; 481-89, 1987.

39. SPIRDUSO WW. Dimensões físicas do envelhecimento. Barueri: Manole, 2005; 310p.

40. VERAS RP. Prevenção de doenças em idosos: os equívocos dos atuais modelos. Cadernos de Saúde Pública, 2012; 28: 1834-1840.

41. VERAS R. Inovação: uma saída para o setor saúde. Revista Brasileira de Geriatria e Gerontologia, 2011; 14(3): 413414. 